www.nature.com/pj

\title{
Microscopic conduction pathways of poly (3-hexylthiophene) nanofibers embedded in polymer film
}

\author{
Polymer Journal (2012) 44, 371-374; doi:10.1038/pj.2012.1; published online 22 February 2012
}

\begin{abstract}
INTRODUCTION
Conjugated conducting polymer nanofibers have recently attracted increasing interest for applications, such as molecular electronics, conductive inks for printable electronics and organic field-effect transistors (OFETs). ${ }^{1-3}$ Polythiophene derivatives including poly(3alkylthiophenes) (P3ATs) are among the most promising conducting polymers, because in addition to high carrier transport based on the $\pi$-conjugated polythiophene chains, the alkyl side chains of P3ATs enable them to be dissolved in common organic solvents. Thus, solvent-soluble P3ATs are suitable for the formation of nanofibers using solutionbased techniques, and in particular, by means of the whisker method using organic solvents. $^{4-7}$
\end{abstract}

An attractive feature of the solution-based process is that different materials can be easily blended to optimize the electrical and mechanical properties according to the device application. OFETs, based on poly(3-hexylthiophene) (P3HT), as a conjugated conducting polymer embedded in the insulating matrix of a polymer film have been reported. These reports suggest that control of phase separation of the conjugated conducting polymer is quite important to form a continuous nanofiber network and exhibit the OFET effect. ${ }^{8-10}$

The electrical percolation behavior in the bulk polymer and on the surfaces of organic electronics containing conducting nanofibers is strongly influenced by the condition of the nanofiber network. Therefore, we have directed our attention to investigation of the microscopic conduction pathways in a nanofiber network of regioregular P3HT (rr-P3HT). Kelvin probe force microscopy (KFM) was employed to investigate the conduction pathways of the nanofiber network. KFM is a useful tool to simultaneously investigate the microscopic topography with atomic force microscopy operation, and the local potential on the surface by the vibrating capacitor method, that is, the Kelvin method. ${ }^{11-13}$ In this study, the whisker method was applied using a solvent mixture of anisole and chloroform $^{5-7}$ to form the P3HT-conducting nanofiber network of rr-P3HT embedded in a poly(methyl methacrylate) (PMMA) matrix as an insulating intermediate.

\section{EXPERIMENTAL PROCEDURE}

rr-P3HT $(\mathrm{Mw}=87000)$ and PMMA $(\mathrm{Mw}=120000)$ were purchased from Aldrich Co. Inc. (St Louis, MO, USA) and used without further purification. Conducting P3HT nanofibers were embedded in PMMA as follows. ${ }^{8} \mathrm{~A} 1-2 \mathrm{wt} \%$ solution of a polymer blend consisting of rr-P3HT and PMMA with a ratio of 1:99 (w/w) was prepared using an organic solvent mixture of anisole and chloroform (50:50, v/v), with stirring at $50{ }^{\circ} \mathrm{C}$. The solution was continuously stirred and gradually cooled from $50^{\circ} \mathrm{C}$ to room temperature at a rate of $20^{\circ} \mathrm{Ch}^{-1}$. A $1 \mathrm{wt} \%$ solution of the polymer blend with only chloroform was also prepared as a reference. ${ }^{8}$

Pt electrodes $(0.6 \mathrm{~mm}$ long) with a gap space of $40 \mu \mathrm{m}$ were fabricated with a bottom-contact configuration by sputter deposition, using a shadow mask on a piece of Si wafer with a 255-nm thermally grown $\mathrm{SiO}_{2}$ layer on top $\left(\mathrm{SiO}_{2} / \mathrm{Si}\right.$; KST World Corp., Fukui, Japan). Thin films of P3HT/PMMA were prepared by spin-coating at 3000 r.p.m. for $30 \mathrm{~s}$ on the $\mathrm{SiO}_{2} / \mathrm{Si}$ substrate with $\mathrm{Pt}$ electrodes, and the residual solvent on the substrate was removed by vacuum drying. For the top-contact configuration, the same $\mathrm{Pt}$ electrodes were fabricated after $\mathrm{P} 3 \mathrm{HT} /$ PMMA thin films were cast on a bare $\mathrm{SiO}_{2} / \mathrm{Si}$ substrate using the same spin-coating conditions.
The thin films of P3HT/PMMA were observed in air with a CP-II scanning probe microscope (Veeco Instruments Inc., Plainview, NY, USA) equipped with a lock-in amplifier, an interface module, an ac voltage source and a dc voltage source for KFM observation. A Pt-coated Si cantilever (OMCL-AC-240TM, Olympus, Tokyo, Japan); spring constant $2 \mathrm{Nm}^{-1}$, resonant frequency $70 \mathrm{kHz}$ ) was used in intermittentcontact mode. A dc-biased sinusoidal voltage of $5 \mathrm{~V}$ at a frequency of approximately $17 \mathrm{kHz}$ was applied between the probe and the sample as the probe electrical signal for the surface potential measurement with the Kelvin method. The electrical conductance of the P3HT/PMMA thin films was measured using a Keithley 236 source measure unit (Keithley Instruments, Inc., Cleveland, $\mathrm{OH}$, USA).

To confirm the influence of the electrode configurations, we performed FET measurements of $\mathrm{P} 3 \mathrm{HT}$ nanofibers without a PMMA matrix formed by the whisker method as reported elsewhere. ${ }^{5-7}$ FETs were composed with $\mathrm{SiO}_{2} / \mathrm{Si}$ substrate, on which P3HT nanofibers were spin-coated and Pt electrodes were sputtered as the source and drain electrodes. The Pt electrodes with the top- and bottom-contact configurations were fabricated using the same methods for the thin films of P3HT/PMMA described above.

The FET measurements were executed in vacuum $\left(<10^{-5}\right.$ Torr $)$ at room temperature, using a combined system of the probe station chamber (ARK-LIPS-LV-2, Nagase Electronic Equipment Service Co., Ltd, Tokyo, Japan), the Keithley 236 source measure unit to measure the source-drain characteristics $I_{\mathrm{SD}}$, and a digital sourcemeter (Keithley 2400, Keithley Instruments, Inc.) to apply the gate voltage $V_{\mathrm{G}}$ from backside gate. 


\section{RESULTS AND DISCUSSION}

The topographic image of the P3HT/PMMA thin film cast from chloroform solution shown in Figure 1a indicates that no nanofibers were formed. In the phase separation process of $\mathrm{P} 3 \mathrm{HT}$ in polymer blends, there has been no report of nanofiber formation when only chloroform is used as a solvent, ${ }^{9}$ which is consistent with the present work. Furthermore, the electrical conductance was less than $10^{-11} \mathrm{~S}$ and the thin film was almost insulative. On the contrary, the topographical image of the thin film formed using the anisole/chloroform solvent mixture (Figure 1b) confirms that conducting P3HT nanofibers were formed in the PMMA matrix. In addition, the thin film of P3HT/PMMA formed with the solvent mixture exhibited much higher conductivity $\left(7.0 \times 10^{-8} \mathrm{~S}\right)$, which was measured for the bottom-contact configuration. These results indicate that the $\mathrm{P} 3 \mathrm{HT}$ nanofibers were well formed in the PMMA matrix using the whisker method with adequate solvent (anisole/ chloroform mixture). The bulk conductivity of the film prepared under these conditions was significant, which indicates that the P3HT nanofibers are electrically connected over the entire bulk film, like a circuit network, and therefore, have a large influence on the film conductivity.

To confirm electrical percolation in the nanofibers of the film, the surface potential of the nanofiber network was measured using KFM, with the application of an external potential bias at $500 \mathrm{mV}$ from the Pt electrodes with bottom-contact configuration using a function generator (DF1906, NF Corp., Yokohama, Japan). Figures $2 \mathrm{a}$ and $\mathrm{b}$ show topographic images of the film surface opposite to the electrodes when applying low $(0 \mathrm{~V})$ and high voltages $(500 \mathrm{mV})$, respectively. Figures $2 \mathrm{c}$ and $\mathrm{d}$ show surface potential images that correspond to Figures $2 \mathrm{a}$ and $\mathrm{b}$, respectively. Nanofibers corresponding to those observed in the topographical images were clearly observed in the surface potential images, and the brightness gradually decreased from high voltage side to the low side. The cross section in the low side (Figure 3) shows that the surface potential of the nanofibers was raised to approximately $470 \mathrm{mV}$, with respect to that of the matrix. These results suggest that the electrical potential applied from the bottom-contact electrodes was percolated to the film surface through the nanofibers, forming a three-dimensional network with the electrical connections, and that the bare nanofibers on the film surface are biased with the externally applied voltage. On the other hand, the higher surface poten-
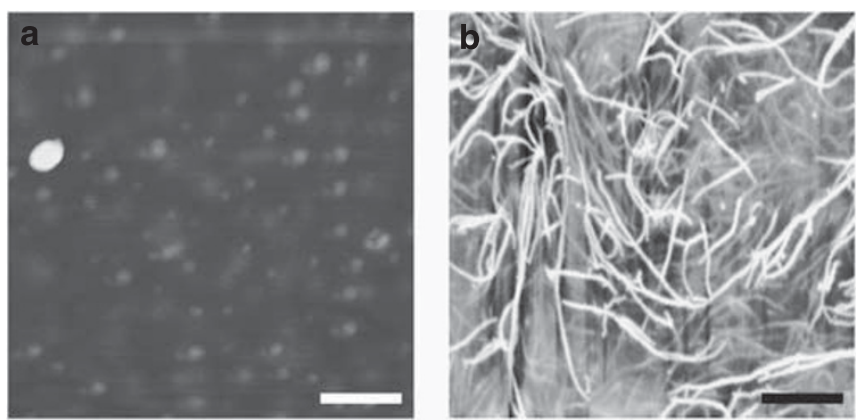

Figure 1 Scanning probe microscope topographical images of P3HT/PMMA thin films produced using (a) only chloroform, and (b) an anisole/chloroform (50:50, v/v) solvent mixture. Scale bars in (a) and (b) are 1 and $5 \mu \mathrm{m}$, respectively. A full color version of this figure is available at Polymer Journal online.
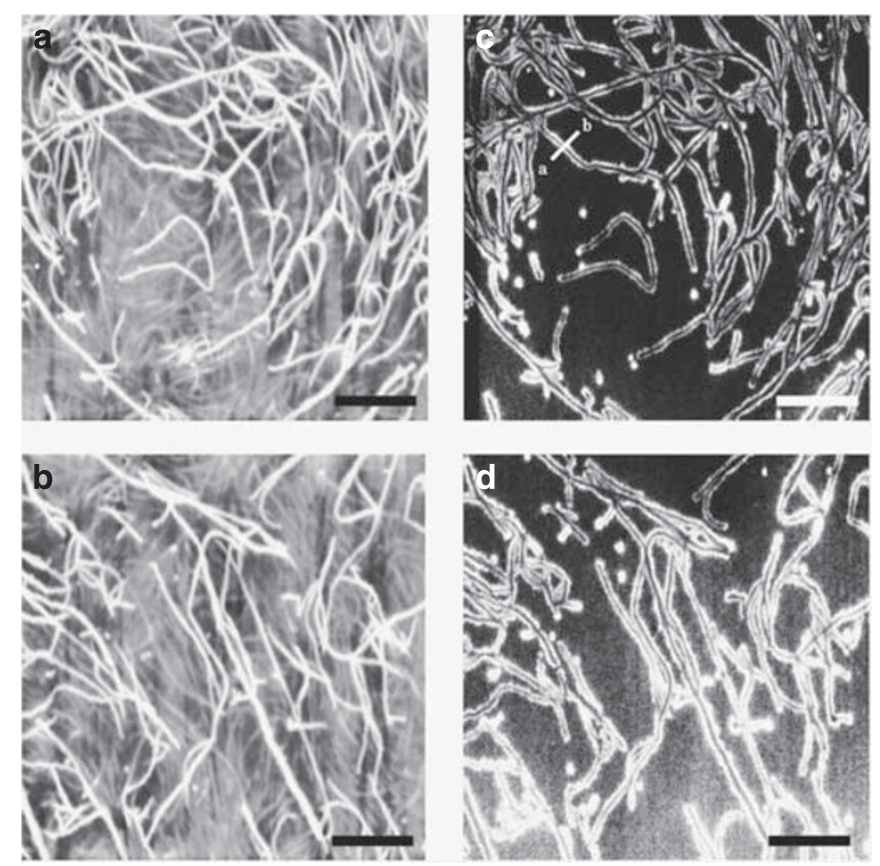

Figure 2 (a, b) Topographical and (c, d) surface potential KFM images of the bottom-contact configuration P3HT/PMMA thin film externally biased at $500 \mathrm{mV}$ (high side in (a) and (c), and low side in (b) and (d)). Two captures were obtained with overlapping. Scale bars are $5 \mu \mathrm{m}$. A full color version of this figure is available at Polymer Journal online.

tial of the matrix in the high side than that of the low side indicates that nanofibers were not bared on the film surface, but embedded in the matrix as shown in the topographical images, influence the surface potential of the matrix. Therefore, the potential difference between the nanofibers and the matrix in the high side decreased to approximately $260 \mathrm{mV}$.

KFM observations were also performed with the top-contact configuration to confirm the conduction pathway from the electrodes to the nanofibers. The topographical images around the electrodes applying low $(0 \mathrm{~V})$ and high voltages $(500 \mathrm{mV})$ are shown in Figures $4 \mathrm{a}$ and $\mathrm{b}$, respectively, and Figures $4 \mathrm{c}$ and $4 \mathrm{~d}$ show the corresponding surface potential images. The electrodes were clearly evident in both the topographical and surface potential images. In contrast, nanofibers within the region $10 \mu \mathrm{m}$ from the electrodes were not clearly evident in the surface potential images, although nanofibers were observed in the entire electrode gap region, as evident in the topographical images. This tendency was independent of the scan direction and scan rate. On the other hand, the film conductivity in the top-contact configuration was $2.9 \times 10^{-8} \mathrm{~S}$, which was comparable to that for the bottom-contact configuration with similar film thickness; therefore, the conduction pathways were connected from one electrode to the counter electrode. These results suggest that the 


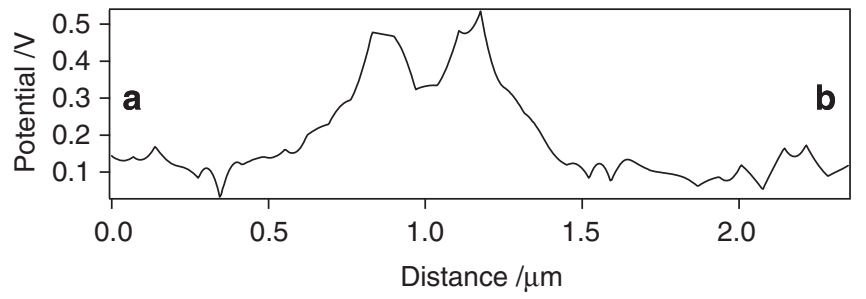

Figure 3 Cross section of the surface potential along the line indicated by $a-b$ in Figure 2c.
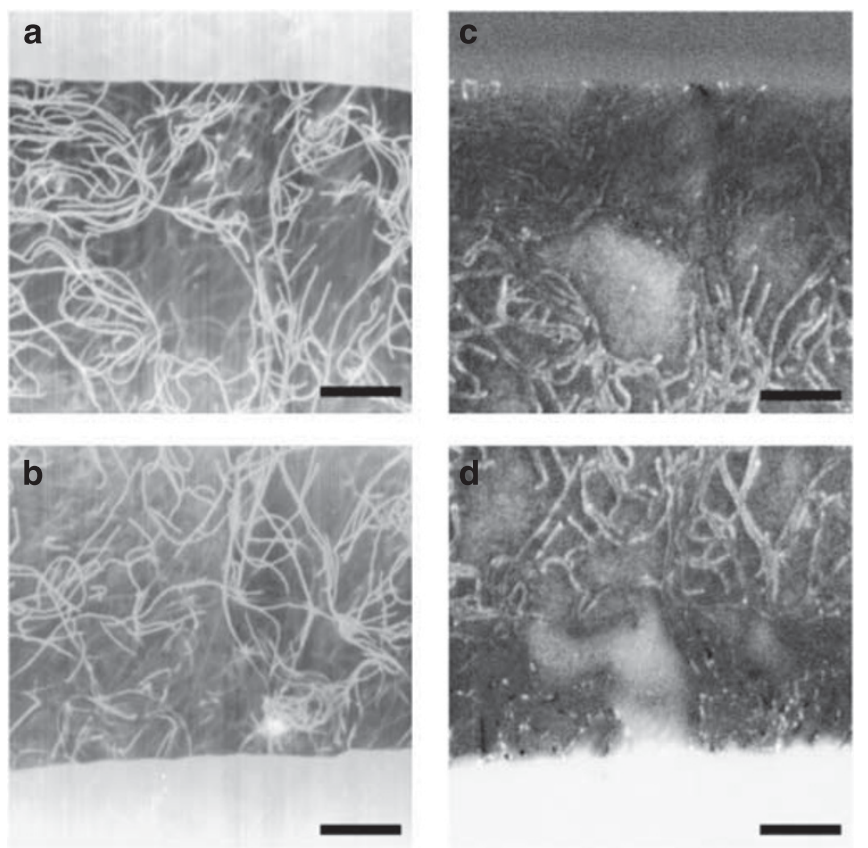

Figure 4 (a, b) Topographical and (c, d) surface potential KFM images of the top-contact configuration P3HT/PMMA thin film externally biased at $500 \mathrm{mV}$ (high side in (a) and (c), and low side in (b) and (d)). Two captures were obtained with overlapping. Scale bars are $5 \mu \mathrm{m}$. A full color version of this figure is available at Polymer Journal online.

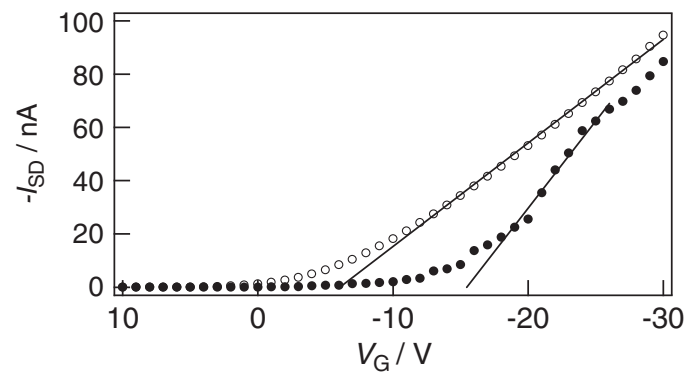

Figure 5 FET transfer characteristics of P3HT nanofibers without a PMMA matrix, where solid lines show the fitting characteristic with a linear relationship. Filled and open circles represent top- and bottom-contact configurations, respectively.

conduction pathways of the film in the top-contact configuration are formed as follows. The electrical flux in the top-contact configuration is at first not parallel to the film surface, but directly perpendicular from the electrode to the interior of the film. And then, the electrical flux leaves for the counter elec- trode with spreading to the bulk, including the film surface apart from the electrodes, along the nanofibers. Therefore, the nanofibers on the film surface apart from the electrodes exhibited the surface potential that reflected the applied voltage in KFM observation and, although the nanofibers on the film surface near the electrodes did not exhibit a surface potential, the conduction pathways were connected between the electrodes via the interior of the film. Furthermore, the nanofibers near the electrodes appeared to be damaged by the sputtering process on the film through processes, such as thermal stress, in the microscopic crystallinity of the nanofibers, and hence, the electrical structures, that is, conjugated chains.

Moreover, as shown in Figure 5, the FET transfer characteristics of P3HT nanofibers without a PMMA matrix were measured with the top- and bottom-contact configurations, applying a source-drain voltage $V_{\mathrm{SD}}$ of $-5 \mathrm{~V}$. The p-type semiconductor characteristic feature in both configurations was observed and the negative threshold gate voltage for the top-contact configuration was higher than that with the bottom-contact configuration. The field-effect mobility $\mu$ for each configuration was calculated from the gradient of linear regime using the following relationship:

$$
\frac{d I_{\mathrm{SD}}}{d V_{\mathrm{G}}}=\frac{W}{L} \mu C_{\mathrm{OX}} V_{\mathrm{SD}}
$$

where $L(=40 \mu \mathrm{m})$ is the spacing of electrodes, $W(=0.6 \mathrm{~mm})$ is the effective width of electrodes, and $C_{\mathrm{ox}}\left(=13.8 \mathrm{nF} \mathrm{cm}^{-2}\right)$ is the capacitance per unit area of the $\mathrm{SiO}_{2}$-insulating ayer with a thickness of $255 \mathrm{~nm}$. The field-effect mobility with the bottomcontact configuration was estimated to be $3.7 \times 10^{-3} \mathrm{~cm}^{2} \mathrm{~V}^{-1} \mathrm{~s}^{-1}$, which was the same order as that reported previously. ${ }^{7}$ On the other hand, the estimated field-effect mobility with the top-contact configuration was $6.3 \times 10^{-3} \mathrm{~cm}^{2} \mathrm{~V}^{-1} \mathrm{~s}^{-1}$, which was higher than that with the bottom-contact configuration; however, this does not indicate that the FET transfer characteristic in the top-contact configuration was higher than that in the bottom-contact configuration, as $I_{\mathrm{SD}}$ in the top-contact configuration approximated to that in the bottom-contact with increasing the negative gate voltage. These differences in the FET transfer characteristics between the top- and bottom-contact configurations indicate that a charge trap ascribed to damaged the nanofibers or substrate was generated by the sputtering process in the top-contact configuration, and is consistent with the KFM measurement of P3HT nanofibers embedded in a PMMA matrix.

In conclusion, the microscopic structures and electrical properties of $\mathrm{P} 3 \mathrm{HT}$ nanofibers embedded in the PMMA matrix were investigated using KFM. This study demonstrated that the conducting nanofibers formed a three-dimensional network with electrical connections, which had a significant influence 
on the electrical properties of the polymer films as conduction pathways.

\section{ACKNOWLEDGEMENTS}

This work was partially supported by a Grant-Aid for Scientific Research (B) (No. 23350111) from the Ministry of Education, Culture, Sports, Science and Technology of Japan.

The microscopic structures and electrical properties of regioregular poly(3-hexylthiophene) (P3HT) nanofibers embedded in a poly(methyl-methacrylate) film as an insulating intermediate by means of whisker method has been investigated using the Kelvin probe force microscopy. This study demonstrated that the conducting P3HT nanofibers formed a three-dimensional network with electrical connections, which had a significant influence on the electrical properties of the polymer films as conduction pathways.

Ken-ichi Yoshida ${ }^{1,2}$, Masahiro Kawasaki ${ }^{1}$, Yuriko Toda ${ }^{1}$, Soko Yamashita ${ }^{1}$ and Takeshi Shimomura ${ }^{1}$
${ }^{1}$ Graduate School of Bio-Applications and Systems Engineering, Tokyo University of Agriculture and Technology, Koganei, Japan and ${ }^{2}$ Materials and Process Development Center, TDK Corporation, Ichikawa, Japan E-mail:simo@cc.tuat.ac.jp

1 Schenning, A. P. \& Meijer, E. W Supramolecular electronics; nanowires from self-assembled $\pi$-conjugated systems. Chem. Commun. 3245-3258 (2005).

2 Aleshin, A. N. Quasi-one-dimensional transport in conducting polymer nanowires. Phys. Solid State 49, 2015-2023 (2007).

3 Briseno, A. L., Mannsfeld, S. C. B., Jenekhe, S. A., Bao, Z. \& Xia, Y. Introducing organic nanowire transistors. Mater. Today 11, 38-47 (2008).

4 Samitsu, S., Shimomura, T. \& Ito, K. Nanofiber preparation by whisker method using solvent-soluble conducting polymers. Thin Solid Films 516, 2478-2486 (2008).

5 Samitsu, S., Shimomura, T., Heike, S., Hashizume, T. \& Ito, K. Effective production of poly(3-alkylthiophene) nanofibers by means of whisker method using anisole solvent: structural, optical, and electrical properties. Macromolecules 41, 8000-8010 (2008).

6 Samitsu, S., Shimomura, T., Heike, S., Hashizume, T. \& Ito, K. Field-effect carrier transport in poly(3-alkylthio- phene) nanofiber networks and isolated nanofibers. Macromolecules 43, 7891-7894 (2010).

7 Shimomura, T., Takahashi, T., Ichimura, Y., Nakagawa, S., Noguchi, K., Heike, S. \& Hasuizume, T. Relationship between structural coherence and intrinsic carrier transport in an isolated poly(3-hexylthiophene) nanofiber. Phys. Rev. B 83, 115314 (2011).

8 Qiu, L., Lim, J. A., Wang, X., Lee, W. H., Hwang, M. \& Cho, K. Versatile use of vertical-phase-separationinduced bilayer structures in organic thin-film transistors. Adv. Mater. 20, 1141-1145 (2008).

9 Qiu, L., Lee, W. H., Wang, H., Kim, J. S., Lim, J. A., Kwak, D., Lee, S. \& Cho, K. Organic thin-film transistors based on polythiophene nanowires embedded in insulating polymers. Adv. Mater. 21, 1349-1353 (2009).

10 Lim, J. A., Kim, J. H., Qiu, L., Lee, W. H., Lee, H. S., Kwak, D. \& Cho, K. Inkjet-printed single-droplet organic transistors based on semiconductor nanowires embedded in insulating polymers. Adv. Funct. Mater. 20, 3292-3297 (2010).

11 Nonnenmacher, T., O'Boyle, M. P. \& Wichramasinghe, H. K. Kelvin probe force microscopy. Appl. Phys. Lett. 58, 2921-2923 (1991).

12 Bürgi, L., Sirringhaus, H. \& Friend, R. H. Noncontact potentiometry of polymer field-effect transistors. Appl. Phys. Lett. 80, 2913-2915 (2002).

13 Miyazaki, T., Kobayashi, K., Ishida, K., Hotta, S., Horiuchi, T., Yamada, H. \& Matsushige, K. Nanoscale electrical properties of molecular films in the vicinity of platinum ultrathin film electrode. Jpn J. Appl. Phys. 42, 4852-4855 (2003). 